\title{
Ising Spin Glasses in a Magnetic Field
}

\author{
J. Houdayer and O. C. Martin \\ LPTMS, bât. 100, Université Paris-Sud, F-91405 Orsay, France.
}

(September 1, 2018)

\begin{abstract}
Ground states of the three dimensional Edwards-Anderson spin glass are computed in the presence of an external magnetic field. Our algorithm is sufficiently powerful for us to treat systems with up to 600 spins. We perform a statistical analysis of how the ground state changes as the field is increased, and reach the conclusion that the spin glass phase at zero temperature does not survive in the presence of any finite field. This is in agreement with the droplet model or scaling predictions, but in sharp disagreement with the mean field picture. For comparison, we also investigate a dilute mean field spin glass model where an Almeida-Thouless line is present.
\end{abstract}

75.50.Lk,75.40.Mg

A long standing controversy in the field of spin glasses concerns the stability of the spin glass phase when a magnetic field is applied. Mean field approaches [1] as well as a number of Monte Carlo simulations [2] suggest that the spin glass phase survives as long as the magnetic field is not too large; an Almeida-Thouless (AT) line [3] then separates this phase from a paramagnetic phase at large fields. On the contrary, scaling approaches [4] 6] predict that the spin glass phase will not survive the application of any finite magnetic field; the system becomes paramagnetic as soon as the external field is non-zero. This controversy remains even though there have been a number of experimental studies (see references in [7,8]); indeed, since it is difficult to reach equilibrium in these systems, many of the experimental measurements may be affected by out of equilibrium artifacts.

More generally, the mean field and scaling approaches differ fundamentally in their view of the nature of large scale excitations. In mean field, the spin glass phase is characterized by numerous nearly degenerate yet widely separated valleys. The energies at the bottom of these valleys are expected to be random [1] as in Derrida's random energy model [9]; also, the characteristic size of the energy gap separating the two lowest energy valleys should be constant, i.e., should not grow with the system size. In the scaling approach, however, there are no such valleys; instead, system-size excitations should have energies which grow as a power of their size.

The question of which approach is "correct" for threedimensional spin glasses remains largely open. Our purpose here is to test numerically the stability of the spin glass phase to the application of an external magnetic field at zero temperature $(T=0)$. To do so, we were led to develop a new and very effective algorithm for computing spin glass ground states. Then, we apply this numerical tool to spin glass models in the presence of a magnetic field. Our main focus is on the three dimensional Edwards-Anderson (EA) model [10] with Gaussian nearest neighbor couplings. For comparison, we also study an appropriately chosen dilute mean field spin glass model. Our conclusion is that in three dimensions the spin glass phase does not survive the introduction of a finite magnetic field, whereas it does survive in mean field models.

Finding ground states - We consider an EA Hamiltonian coupling nearest neighbor spins on a threedimensional cubic lattice with periodic boundary conditions:

$$
H_{J}\left(\left\{S_{i}\right\}\right)=-\sum_{<i j>} J_{i j} S_{i} S_{j}-B \sum_{i} S_{i} .
$$

The first sum is over all bonds of the lattice, i.e., over all nearest neighbor spin pairs; $B$ is the external magnetic field, and the $J_{i j}$ 's are quenched couplings. We take these couplings to be independent random variables with a Gaussian distribution of zero mean and unit variance. The $J_{i j}$ 's generate quenched disorder, and since the sign of these couplings varies, they introduce frustration.

Finding the ground states of this system is NPhard [11]. Not surprisingly then, even the state of the art algorithms [12] for finding ground states are very slow and are not able to effectively go beyond $5 \times 5 \times 5$ lattices. Because of this, studies of spin glass ground states have often [13] used "heuristic" algorithms which only find the ground state with a high probability. For our study, we have developed a new heuristic algorithm which is based on iteratively optimizing the spin configurations on all scales using a recursive genetic algorithm. It is not guaranteed to find the lowest state, but we have performed checks to measure its power and reliability [14]. In particular, we have used the magnetic field to derive a self-consistency check as follows. We start with a large magnetic field and compute the putative ground state. This ground state is then used as a starting point for our iterative improvement algorithm at a lower value of the field. We can then check for consistency by going to negative values of the field and seeing whether the states found are exactly the same (up to a global flip of the spins) as those obtained at positive values of the field. If they are not, we reinitiate our genetic algorithm with a larger population. Using this approach, we estimate the error rate for finding the true ground state to be less than 1 in $10^{5}$. 
Effect of the field - For each lattice size and realization of the disorder variables $J_{i j}$ 's, we have determined the ground state configuration as a function of the magnetic field $B$. For large $B$, nearly all spins are parallel to the field. As the field is decreased continuously, the ground state changes at a series of values of $B$; these values can be thought of as comprising a discrete and finite "spectrum" for each instance. For a given point of this spectrum, let $s$ be the number (or volume) of spins which are flipped when going from one ground state to the next. Since the $J_{i j}$ 's are continuous, these $s$ spins form a connected cluster with probability 1 . (Note that the ground state is generically non degenerate as soon as $B \neq 0$.) We focus our study on the statistical properties of $s$ as a function of $B$. We have also measured the total magnetization associated with these clusters of $s$ spins.

Before giving our results, let us describe qualitatively what is predicted to occur in the scaling and mean field pictures. Let us begin with $B=0$. In the scaling picture, it is useful to introduce the notion of a droplet [6]. For each site and each length $l$, consider the excitation of volume $O\left(l^{3}\right)$ which has the lowest energy and contains the spin at that site. This excitation corresponds to flipping a connected cluster of spins and is called a droplet. In the scaling approach, hereafter called the droplet picture, the energy of a droplet scales as $l^{\theta}$ as its "diameter" $l$ grows. Now take the limit where the droplet spans a finite fraction of the whole lattice; then its energy is predicted to grow as $N^{\theta / 3}$ in three dimensions if $N$ is the total number of spins. This is to be contrasted with what happens in the mean field picture. In analogy with what occurs in the Sherrington-Kirkpatrick model, the mean field picture suggests that there are system-size excitations whose energy does not grow with $N$, so effectively $\theta=0$.

Now apply an infinitesimal field $B$. The magnetic term in the energy can overcome the interfacial energy of a droplet of volume $s$ and force it to flip. This will occur when the magnetic energy, of order $s^{1 / 2} B$, becomes larger that the interfacial energy of order $s^{\theta / 3}$. From such a scaling, it is apparent that system size droplets are the most unstable and flip for field intensities

$$
B_{\min }=N^{\theta / 3-1 / 2} .
$$

(In the case of the mean field picture, the same reasoning applies, but with $\theta=0$.) Both pictures thus predict the appearance of system size events $(s=O(N))$ in the spectrum near $B=0$, albeit at different scales in $B$. Since $\theta$ is small, $(\theta \approx 0.2[5])$ the predictions for $B_{\min }$ in the two different pictures are difficult to distinguish numerically.

Interestingly, the situation is much more clear-cut when $B$ is finite rather than infinitesimal because the two pictures then differ dramatically. In that regime of magnetic field, there is a non-zero mean magnetization per site which is proportional to $B$. In the droplet picture, this magnetization makes it unfavorable to flip large scale clusters because the magnetic energy term now grows as $s B^{2}$ and is of the wrong sign. Thus in this approach, the sizes $s$ appearing in the spectrum are small (finite) as soon as $B$ is non-zero. The field has introduced a length scale beyond which there are no low energy excitations; the system is then paramagnetic. Thus the spin glass phase does not survive when $B \neq 0$, and there is no AT line. On the contrary, in the mean field picture, the energy landscape continues to have many coexisting valleys even at finite values of $B$. Furthermore, in this picture, these valleys have random and rather independent energies at their bottoms. Then changing the magnetic field should lead to level crossing of these valleys, so that the ground state is expected to jump chaotically from one valley to another at high frequency, generating events with $s=O(N)$ even when there is a mean magnetization.

Finite size scaling - Let us see what happens in reality. Our approach is based on the statistical analysis of our data taken in the ensemble where the $J_{i j}$ 's are Gaussian random variables. Consider an $L \times L \times L$ lattice of $N$ spins and a small interval $[B, B+\Delta B]$. In the limit $\Delta B \rightarrow 0$, the frequency with which the ground states change in this interval is proportional to $\Delta B$. Since the two pictures just discussed differ in their predictions for events satisfying $s=O(N)$, we focus on the "rate" $r(N, B, x)$ (per $\Delta B$ and per $\Delta x)$ at which a ground state is changed by flipping $s=x N$ spins. To determine $r(N, B, x)$, we find for each instance the ground states at regularly spaced values of $B$. For each interval, we find the set of spins which have been flipped and find the corresponding clusters defined as the connected components of that set. We then cumulate the sizes $s$ of the events in a histogram, and average over $10^{3}$ to $10^{5}$ randomly generated instances for lattice sizes $L=3,4,5,6$ and 8 . Then the estimator for $r(N, B, x)$ is obtained by summing this histogram for $s \in[(x-\Delta x / 2) N,(x+\Delta x / 2) N]$ and dividing this sum by $\Delta B \Delta x$. We take $\Delta B=0.1$, a small enough value so that the juxtaposition of several events is not mistaken for one large event; we also set $\Delta x=0.1$. In view of the qualitative discussion of valleys given in the previous section the signature of the existence of the AT line is the survival of $r(N, B, x)$ at finite values of $x$ and $B$ as $N \rightarrow \infty$. More precisely, for any fixed $x>0$ and $B \neq 0$, the droplet model predicts $r(N, B, x) \rightarrow 0$ as $N \rightarrow \infty$, whereas the mean field picture predicts $r(N, B, x)$ does not tend to zero as $N \rightarrow \infty$ (as long as $B$ is not too large).

The data for $r(N, B, x)$ as a function of $B$ for different lattice sizes are displayed in Figure1 1 in the case $x=0.15$. Other values of $x$ give qualitatively similar results: the curves go to zero at large fields, and as $N$ grows the curves cross at smaller and smaller values of $B$. Furthermore it seems that for fixed $B, r(N, B, x) \rightarrow 0$ as 


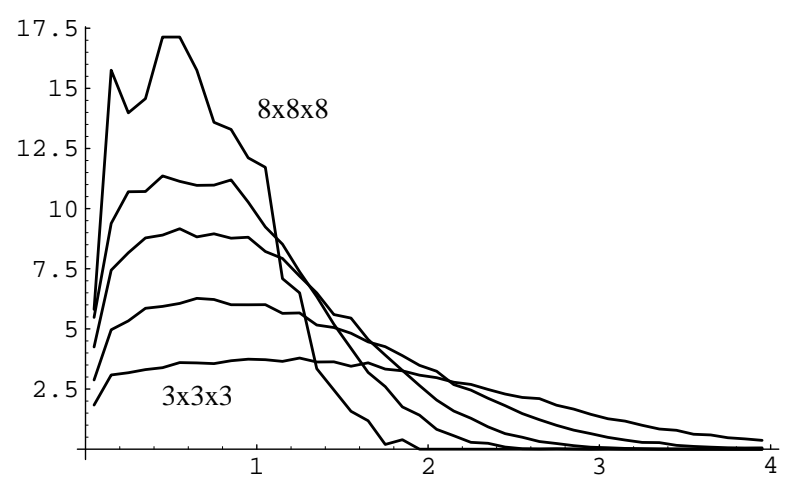

FIG. 1. $r(N, B, 0.15)$ as a function of $B$ for the EA model with lattice sizes $3^{3}, 4^{3}, 5^{3}, 6^{3}$ and $8^{3}$.

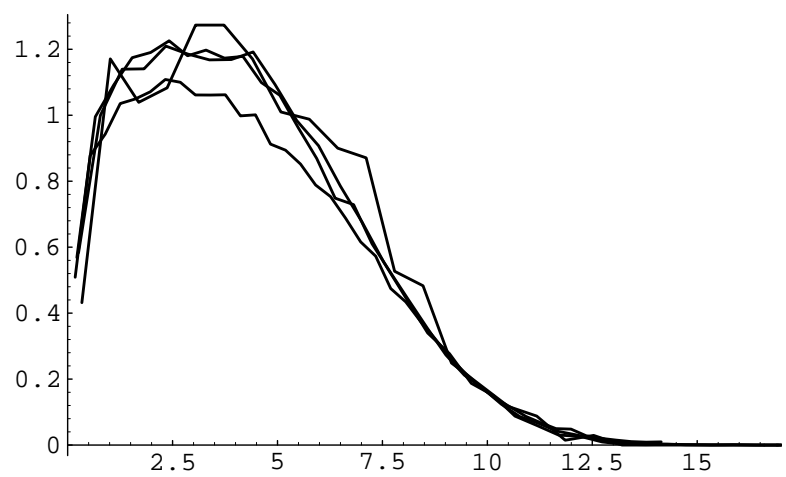

FIG. 2. $r(N, B, 0.15) / N^{\mu}$ as a function of $B N^{\lambda}$ for the EA model with lattice sizes $4^{3}, 5^{3}, 6^{3}$ and $8^{3}$. We find $\mu=0.42$ and $\lambda=0.31$.

$N \rightarrow \infty$. To put such an extrapolation on a sound footing, we apply finite size scaling, searching for a way to collapse all of the curves onto one-another. For $N \geq 4^{3}$, we were able to do this by using a power law scaling:

$$
r(N, B, x) \approx N^{\mu} R_{x}\left(B N^{\lambda}\right) .
$$

This is shown in Figure 2 for $x=0.15$, but the data collapse occurs also for the other values of $x$, and with the same exponents. The displayed scaling function falls to zero rapidly, seemingly faster than any power, showing that large scale events become arbitrarily rare with system size if the field is non-zero, contradicting the mean field picture. These results indicate that the spin glass phase does not survive the application of a finite magnetic field at zero temperature, in agreement with the droplet picture prediction.

Comparison to a mean field model — The nonexistence of a spin glass phase at $T=0$ and $B \neq 0$ is not surprising from the point of view of the droplet picture. Were one to try to defend the mean field picture of the spin glass phase persisting at finite $B$, one would have

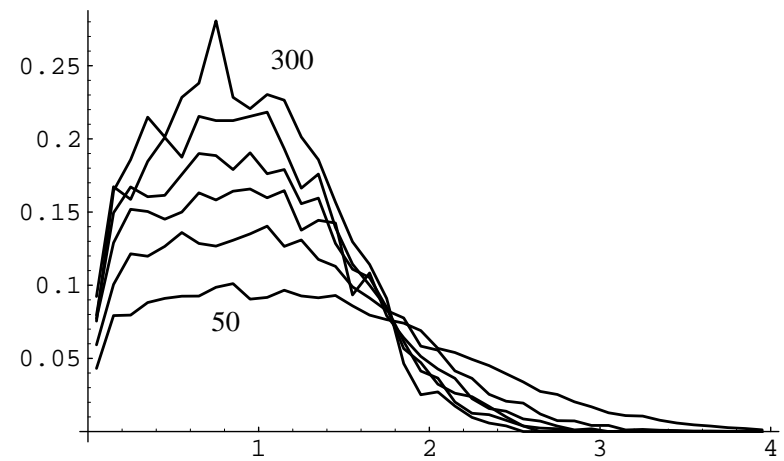

FIG. 3. $r(N, B, 0.15)$ as a function of $B$ for the mean field model with system sizes 50, 100, 250, 200, 250 and 300.

to argue that the finite size scaling found in Figure 2 is not perfect, and that larger system sizes might begin to show that the correct interpretation of the data is different. Thus it is useful to check that our proposed method of extrapolation is sensible when considering a different model where we know that the mean field picture is valid. To do so, we consider a dilute spin glass where there is no space or geometry, but which is otherwise as close as possible to the 3-dimensional EA model. Thus we take the spins to be connected at random while maintaining the connectivity fixed at 6 for each spin. This model 15] is not very different from a Viana-Bray model [16]. The $J_{i j}$ couplings on the bonds which are present are taken to have the same Gaussian distribution as in the lattice model. The motivation for using this model rather than the Viana-Bray dilute spin glass follows from the fact that the Euclidean (lattice) model and this mean field model have the same Cayley tree approximation.

We have analyzed this dilute spin glass model in the same way as we analyzed the 3 -dimensional lattice model. We find that the raw data shows a significant difference with the Euclidean case: the different curves all cross near the same point (see Figure 3). Performing finite size scaling analysis, we find that the curves for different $N$ do not superpose if we scale the field $B$, but do superpose on the left of the crossing point if we use the scaling

$$
r(N, B, x) \approx \sqrt{N} Q_{x}(B),
$$

and this is true for all $x<0.5$.

The limiting large $N$ curve $Q_{x}$ falls to zero at a critical field $B_{x}^{*}$ which is identified by the crossing of the curves on Figure 3. This is supported by a finite size scaling analysis at large $N$ : as shown in Figure 4 , the different curves superpose when we plot $r(N, B, x)$ as a function of $\sqrt{N}\left(B-B_{x}^{*}\right)$. It is intuitively clear and we find that $B_{x}^{*}$ decreases as $x$ grows; let the largest value of $B_{x}^{*}$ be $B_{c}$ (we find $B_{c} \approx 2.1$ ), beyond which there are no longer any events with $s=O(N)$. $B_{c}$ can be interpreted as 


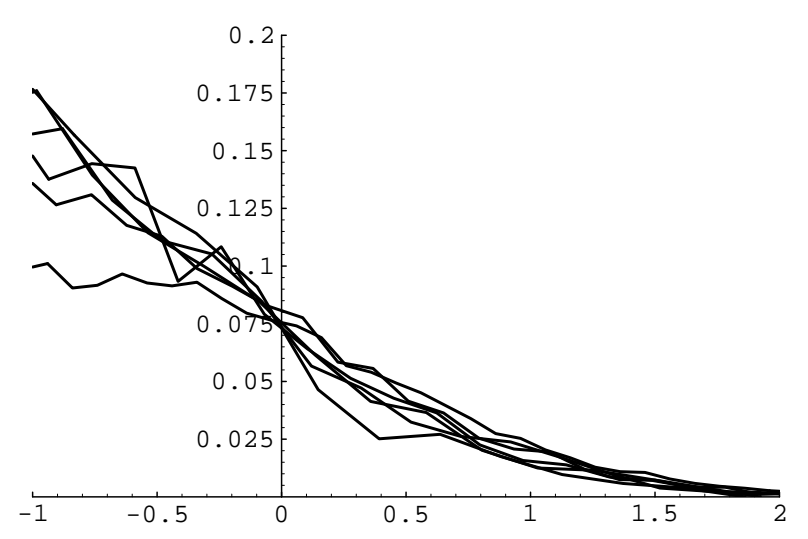

FIG. 4. $r(N, B, 0.15)$ as a function of $\sqrt{N}\left(B-B_{0.15}^{*}\right)$ for the mean field model with system sizes 50, 100, 250, 200, 250 and 300 . We find $B_{0.15}^{*}=1.79$.

the crossing of the AT line with the zero temperature axis, so for $B<B_{c}$ the system is in the spin glass phase and for $B>B_{c}$ it becomes paramagnetic. (Note: in the Sherrington-Kirkpatrick model, the AT line goes to $B=$ $\infty$ as $T \rightarrow 0$. For our dilute spin glass $B_{c}(T)$ remains bounded as $T \rightarrow 0$ because the model's connectivity is finite.)

Discussion - The picture to draw from our results is that in the presence of a finite field, the 3-dimensional EA model behaves at zero temperature like a paramagnet, as predicted by the droplet model. The spin glass phase is thus destroyed by any finite magnetic field. This conclusion is in agreement with recent experimental measurements 17]. To interpret this result, it is natural to invoke the usual argument from the droplet model: once the system has a mean magnetization $m>0$, it becomes nearly impossible to find large scale objects to flip which have negative magnetization and whose interfacial energy is not large. Nevertheless, we do not claim that all of our results are explained by the droplet model. As discussed before, the characteristic field intensity at which system size excitations arise is given by Equation 2. Comparing with Equation 3 leads to $\lambda=1 / 2-\theta / 3$. Since we find $\lambda \approx 0.31$, this would correspond to $\theta \approx 0.57$ which is not compatible with the commonly accepted value $\theta \approx 0.2$.

We have also investigated a dilute spin glass model which is the natural mean field model for the 3dimensional EA model. Although a finite magnetic field gives rise to a finite magnetization, there is no associated length scale, and the model maintains a spin glass phase up to some maximum field intensity $B_{c}$. If we take at face value the droplet model interpretation given above, we can say that since this mean field model has no space embedding, large clusters are never compact, and because of that, it becomes possible to find clusters of negative magnetization whose interfacial energy is small. Our study of this model also shows that it is not the infinite connectivity but rather the absence of geometry which allows the spin glass phase to survive in the presence of a magnetic field.

Acknowledgements - We thank Jean Philippe Bouchaud for his very useful comments. J. H. acknowledges a fellowship from the MENESR, and O.C.M. acknowledges support from the Institut Universitaire de France. The LPTMS is an Unite de Recherche de l'Université Paris XI associée au CNRS.

[1] M. Mézard, G. Parisi, and M. A. Virasoro, Spin-Glass Theory and Beyond, Vol. 9 of Lecture Notes in Physics (World Scientific, Singapore, 1987).

[2] E. Marinari, C. Naitza, and F. Zuliani, , condmat/9802224.

[3] J. R. L. de Almeida and D. J. Thouless, J. Phys. A 11, 983 (1978).

[4] W. L. McMillan, Phys. Rev. B 31, 340 (1985).

[5] A. J. Bray and M. A. Moore, in Heidelberg Colloquium on Glassy Dynamics, Vol. 275 of Lecture Notes in Physics, edited by J. L. van Hemmen and I. Morgenstern (Springer, Berlin, 1986), pp. 121-153.

[6] D. S. Fisher and D. A. Huse, Phys. Rev. B 38, 386 (1988).

[7] K. Binder and A. P. Young, Rev. Mod. Phys. 58, 801 (1986).

[8] K. H. Fischer and J. A. Hertz, Spin-Glasses, Vol. 1 of Cambridge Studies in Magnetism (Cambridge University Press, Cambridge, 1991).

[9] B. Derrida, Phys. Rev. B 24, 2613 (1981).

[10] S. F. Edwards and P. W. Anderson, J. Phys. F 5, 965 (1975).

[11] C. H. Papadimitriou and K. Steiglitz, Combinatorial Optimization: Algorithms and Complexity (Prentice Hall, Englewood Cliffs, NJ, 1982).

[12] C. de Simone et al., J. Stat. Phys. 84, 1363 (1996).

[13] A. Hartmann, To appear in Phys. Rev. E (1998), condmat/9806114.

[14] J. Houdayer and O. C. Martin, (preprint), submitted.

[15] C. de Dominicis and Y. Goldschmidt, J. Phys. A Lett. 22, L775 (1989).

[16] L. Viana and A. J. Bray, J. Phys. C 18, 3037 (1985).

[17] J. Mattsson et al., Phys. Rev. Lett. 74, 4305 (1995). 